\title{
Techniques and short-term outcomes for total minimally invasive Ivor Lewis esophageal resection in distal esophageal and gastroesophageal junction cancers: pooled data from six European centers
}

\author{
Jennifer Straatman ${ }^{1,7} \cdot$ Nicole van der Wielen ${ }^{1}$ Grard A. P. Nieuwenhuijzen ${ }^{2}$. \\ Camiel Rosman $^{3} \cdot$ Josep Roig $^{4} \cdot$ Joris J. G. Scheepers ${ }^{5} \cdot$ Miguel A. Cuesta ${ }^{1}$. \\ Misha D. P. Luyer ${ }^{2}$. Mark I. van Berge Henegouwen ${ }^{6} \cdot$ Frans van Workum $^{3}$. \\ Suzanne S. Gisbertz ${ }^{6} \cdot$ Donald L. van der Peet ${ }^{1}$
}

Received: 9 December 2015/ Accepted: 9 April 2016/Published online: 29 April 2016

(C) The Author(s) 2016. This article is published with open access at Springerlink.com

\begin{abstract}
Introduction Esophagectomy for cancer can be performed in a two-stage procedure with an intrathoracic anastomosis: the Ivor Lewis esophagectomy. A growing incidence of distal and gastroesophageal junction adenocarcinomas and increasing use of minimally invasive techniques have prompted interest in this procedure. The aim of this study was to assess short-term results of minimally invasive Ivor Lewis esophagectomy (MIE-IL).

Methods A retrospective cohort study was performed from June 2007 until September 2014, including patients that underwent MIE-IL for distal esophageal and gastroesophageal junction cancer in six different hospitals in the Netherlands and Spain. Data were collected with regard to operative techniques, pathology and postoperative complications.
\end{abstract}

Jennifer Straatman

je.straatman@vumc.nl

1 Department of Gastrointestinal Surgery, VU University Medical Center, Amsterdam, The Netherlands

2 Department of Gastrointestinal Surgery, Catharina Hospital, Eindhoven, The Netherlands

3 Department of Gastrointestinal Surgery, Canisius Wilhelmina Hospital, Nijmegen, The Netherlands

4 Department of Gastrointestinal Surgery, Hospital Universitari Dr. Josep Trueta, Girona, Spain

5 Department of Gastrointestinal Surgery, Reinier de Graaf Hospital, Delft, The Netherlands

6 Department of Gastrointestinal Surgery, Academic Medical Center, Amsterdam, The Netherlands

7 Department of Surgery, VU University Medical Center, De Boelelaan 1117, $1081 \mathrm{HV}$ Amsterdam, The Netherlands
Results In total, 282 patients underwent MIE-IL, of which $90.2 \%$ received neoadjuvant therapy. Anastomotic leakage was observed in 43 patients (15.2\%), of whom 13 patients $(4.6 \%)$ had empyema, necessitating thoracotomy for decortication. With an aggressive treatment of complications, the 30-day and in-hospital mortality rate was $2.1 \%$. An R0-resection was obtained in $92.5 \%$ of the patients. After neoadjuvant therapy, $20.1 \%$ of patients had a complete response.

Conclusions Minimally invasive Ivor Lewis esophagectomy for distal esophageal and gastroesophageal junction adenocarcinomas is an upcoming approach for reducing morbidity caused by laparotomy and thoracotomy. Anastomotic leakage rate is still high possibly due to technical diversity of anastomotic techniques, and a high percentage of patients treated by neoadjuvant chemoradiotherapy. An aggressive approach to complications leads to a low mortality of $2.1 \%$. Further improvement and standardization in the anastomotic technique are needed in order to perform a safe intrathoracic anastomosis.

Keywords Esophageal cancer · Esophagectomy · Minimally invasive · Ivor Lewis · Intrathoracic anastomosis

In 1946, a standardized approach to esophageal resection for carcinoma of the middle third of the esophagus was introduced by Ivor Lewis [1]. This approach involved a two-stage procedure that included a laparotomy with lymphadenectomy of the celiac trunk and formation of a gastric conduit and, 1-2 weeks later, a right thoracotomy with esophageal resection, peri-esophageal and subcarinal lymphadenectomy followed by intrathoracic anastomosis. Risk of anastomotic leakage in the thorax with its potential 
fatal sequelae-such as empyema-resulted in the development of the three-stage approach by McKeown, with a cervical anastomosis [2]. In case of leakage, a cervical fistula remained a manageable complication [3]. While randomized evidence is limited, comparative studies suggest that cervical anastomosis is associated with less serious complications, but with more anastomotic leakage, stenosis and recurrent laryngeal nerve injuries [4]. This cervical morbidity in combination with the increased incidence of distal esophageal and gastroesophageal junction adenocarcinomas and lower postoperative morbidity after minimally invasive esophagectomy (MIE) has induced renewed interest in the two-stage procedure with an intrathoracic anastomosis [5].

In recent years, two important developments have been introduced in esophageal surgery, i.e., the systematic use of neoadjuvant treatment (chemotherapy or chemoradiation) and the implementation of minimally invasive esophagectomy (MIE) [5, 6]. Neoadjuvant treatment significantly increases 5-year survival of patients with esophageal cancer in both squamous cell and adenocarcinomas [6]. In addition, minimally invasive esophagectomy is increasingly being implemented in order to reduce postoperative respiratory complications and enhance the quality of life by avoiding a right thoracotomy and laparotomy [7-11].

Aim of this study was to assess and describe the pooled results of six European institutions performing a total minimally invasive Ivor Lewis esophagectomy (MIE-IL) with respect to technique and the resulting short-term postoperative outcomes such as morbidity and mortality and radicality of resection.

\section{Materials and methods}

A retrospective cohort study was performed of 282 consecutive patients, who underwent a total MIE-IL for distal esophageal cancer $(n=160)$ and gastroesophageal junction cancer ( $n=122$ patients) since the introduction of this approach in June 2007 [12]. Patients originated from Girona, Spain and five centers in the Netherlands (VU University medical center and Academic Medical Center, Amsterdam; Canisius Wilhelmina hospital in Nijmegen, Catharina hospital in Eindhoven and Reinier de Graaf hospital in Delft). Data were recorded from 2007 in one center in Girona. In two centers in the Netherlands, data were recorded from 2010 and in three other Dutch centers data were recorded from 2012.

All hospitals performed at least over 20 esophagectomies per year. Two hospitals were academic hospitals, being the VU University medical center and Academic Medical Center, both located in Amsterdam. The other participating hospitals are all teaching hospitals.
A database was constructed with preoperative, intraoperative and postoperative data of these patients. The indices used were age, gender, type and location of the tumor, preoperative assessment, administration of neoadjuvant therapy, operative technique (thoracoscopic approach in prone position or left lateral decubitus position), anastomotic technique, conversions, (y)pTNM, R0 resections, circumferential resection margins, lymph node yield, postoperative complications, duration of hospital stay, intensive care (IC) stay and 30-day and in-hospital mortality.

\section{Statistical analysis}

Continuous data were described using mean and standard deviation or median and interquartile range as appropriate for normal and non-normal distributed data. Analysis was performed with Student's $T$ test for two samples or ANOVA with post hoc analysis for $k$ samples for normal distributions, and for non-normal distributions, the MannWhitney $U$ test was applied or the Kruskal-Wallis test for $k$ samples. For dichotomous and categorical data, frequencies were displayed. Analysis was performed with Chi-square and regression techniques.

\section{Definitions}

Anastomotic leakage was defined as a full-thickness defect involving the esophagus, anastomosis or the gastric conduit and graded according to severity, in concordance with the report on standardization of data collections for complications associated with esophagectomy [13]. A grade I anastomotic leak concerned patients with a local defect, which did not require invasive therapy. A grade II anastomotic leak concerned those patients requiring interventional, but not surgical therapy (i.e., percutaneous drainage, placement of an endoscopic stent). A grade III anastomotic leak without thoracic empyema concerns those patients requiring surgical treatment such as thoracoscopic debridement, mediastinal drainage and placement of a stent, and grade IV leakage concerns established thoracic empyema requiring thoracotomy for decortication, drainage of the leakage, reconstruction of the anastomosis or resection of the necrotic gastric tube. Pulmonary complications included pneumonia as diagnosed on chest X-ray, sputum culture or CT scan and postoperative acute respiratory distress syndrome (ARDS). Wound infections were diagnosed upon positive culture or evident purulent drainage from the surgical wounds. Cardiovascular complications included atrial fibrillation, infarction or heart failure as seen on electrocardiogram (ECG), ultrasound and in laboratory findings. All complications were additionally 
recorded and graded according to the Clavien-Dindo classification [14].

Patients were examined clinically daily; additional examinations were performed on indication. If indicated, examination of the anastomosis consisted of computed tomography scan with oral contrast and/or endoscopy.

Postoperative monitoring for complications was similar in all included hospitals, consisting of daily assessment of clinical parameters. Upon clinical deterioration (i.e., fever, pain, tachycardia, SIRS and ileus), additional laboratory assessment and imaging were performed, consisting of CT scan imaging with oral contrast or endoscopy. Treatment was initiated immediately upon diagnosis of complications.

\section{Operative technique}

Minimally invasive Ivor Lewis esophagectomy starts with a laparoscopic approach, in which lymphadenectomy of lymph node stations $1-4,7-9,11$ and in some centers 12 according to the 10th edition of the JSED classification is performed [15]. Subsequently, a gastric tube is created, and a dissection of the lower paraesophageal lymph nodes via the hiatus could be performed. After changing to prone or lateral decubitus position, thoracoscopy is performed. In prone position, double lung ventilation and a pneumothorax of 6-8 mmHg are maintained with insufflation of $\mathrm{CO}_{2}$ with a maximum pressure of $8 \mathrm{mmHg}$. In left lateral decubitus position, the right lung is blocked and solely the left lung is ventilated. A lymphadenectomy is performed of peri-esophageal, bronchial and subcarinal lymph nodes (lymph node stations 107-111), depending on the center or on indication stations 105, 106tbL, and 106recL and 106 recR according to the 10th edition of the JSED classification [16]. The esophagus is divided proximal of the arcus of the azygos vein, and before or after extraction of the specimen through a small thoracotomy, an anastomosis between the proximal esophagus and the gastric tube is performed [17].

The anastomosis is performed with different methods in the different participating centers. Some centers perform an end-to-side anastomosis using a circular stapler of 25 or $28 \mathrm{~mm}$, including the 25 -mm Orvil type ${ }^{\circledR}$. Others perform a side-to-side anastomosis with a linear endostapler and closure of the defect with a V-Loc ${ }^{\circledR}$ suture. In many cases this is followed by an omental wrap to protect the anastomosis which could reduce the sequelae of an anastomotic leakage [18]. Differences in the use of anastomotic technique reflect local expertise and the search for optimal techniques as described elsewhere [18].

In prone position, the use of a glove adhesive to the protection ring of the wound or a single port permits continuity of insufflation during formation of the anastomosis.

\section{Results}

From June 2007 until September 2014, 282 patients in six different hospitals underwent a total MIE-IL for esophageal cancer. Each participating center performed over 20 esophagectomies per year, with two surgeons performing the procedure in each center. Pertaining characteristics of the 282 patients and peri-operative data are presented in Tables 1 and 2. In total, $90.2 \%$ of patients received neoadjuvant therapy, usually consisting of chemoradiotherapy according to the CROSS protocol [6].

The majority of patients $(89.0 \%)$ were operated in prone position. An end-to-side anastomosis was performed with either a $25-\mathrm{mm}$ stapler in $56.3 \%$ or a $28-\mathrm{mm}$ stapler in $13.7 \%$ of patients. A side-to-side anastomotic technique was performed in $29.4 \%$. An end-to-end hand-sewn anastomosis was performed in $0.7 \%$.

\section{Complications}

In this cohort, four intraoperative complications were recorded. In one patient, a lesion of the splenic artery necessitated a laparoscopic splenectomy. In another patient, part of the balloon of the selective tracheal tube migrated into the right bronchus, which had to be removed by bronchoscopy. In two patients, the operation was complicated by an aortic lesion, in one patient the stapling device perforated the aortic arch, the other occurred during esophageal dissection. In both cases, the operation was converted to a thoracotomy in prone position, and both

Table 1 Baseline characteristics of patients that underwent minimally invasive Ivor Lewis esophagectomy

\begin{tabular}{llr}
\hline Baseline characteristics & $N(282)$ & $\%$ \\
\hline Gender & & \\
Male & 218 & 77.3 \\
Female & 64 & 22.7 \\
Age (mean \pm SD) & $62.8 \pm 8.6$ & \\
Tumor type & 2 & \\
Adenocarcinoma & 229 & 81.2 \\
Squamous cell carcinoma & 29 & 10.3 \\
Adenosquamous cell carcinoma & 24 & 8.5 \\
Tumor location (cm) (mean \pm SD) & $36.2 \pm 3.7$ & \\
Neoadjuvant therapy & & \\
None & 33 & 11.3 \\
Chemoradiotherapy & 233 & 82.6 \\
Chemotherapy & 16 & 5.7 \\
Radiotherapy & 1 & 0.4 \\
\hline
\end{tabular}

Frequencies and percentages are depicted for categorical data, and mean and standard deviations (SD) are depicted for continuous data, after checking for a normal distribution 
Table 2 Peri-operative data for minimally invasive Ivor Lewis esophagectomy

\begin{tabular}{|c|c|c|}
\hline Operative data & $N(282)$ & $\%$ \\
\hline Type of surgery positioning & $\begin{array}{l}\text { Minimally invasive Ivor Lewis } \\
\text { esophagectomy }\end{array}$ & \\
\hline Prone position & 251 & 89.0 \\
\hline Lateral decubitus & 31 & 11.0 \\
\hline Peri-operative blood loss (ml) & $242 \pm 228$ & \\
\hline Duration of surgery (min) & $333 \pm 98$ & \\
\hline \multicolumn{3}{|l|}{ Pathology } \\
\hline T0 & 58 & 24.8 \\
\hline $\mathrm{T} 1$ & 48 & 20.5 \\
\hline $\mathrm{T} 2$ & 31 & 3.2 \\
\hline $\mathrm{T} 3$ & 94 & 40.2 \\
\hline $\mathrm{T} 4$ & 3 & 1.3 \\
\hline No & 140 & 59.3 \\
\hline N1 & 48 & 20.3 \\
\hline $\mathrm{N} 2$ & 34 & 14.2 \\
\hline N3 & 14 & 5.9 \\
\hline Number of lymph nodes & $22.9 \pm 9.7$ & \\
\hline $\mathrm{R} 0$ resection & 185 & 92.5 \\
\hline Complete regression & 30 & 20.1 \\
\hline \multicolumn{3}{|l|}{ Hospital stay [median (IQR)]* } \\
\hline Overall & $12(9-24)$ & \\
\hline Uncomplicated & $10(8-13)$ & \\
\hline Complicated & $23(12-41)$ & \\
\hline \multicolumn{3}{|l|}{ ICU stay [median (IQR)]* } \\
\hline Overall & $2(1-5)$ & \\
\hline Uncomplicated & $2(1-3)$ & \\
\hline Complicated & $3(1-9)$ & \\
\hline Complications & 123 & 43.6 \\
\hline Mortality (30-days) & 6 & 2.1 \\
\hline
\end{tabular}

patients had an uncomplicated postoperative recovery. Conversion to an open procedure, for oncological reasons or extensive pleural adhesions, occurred in five patients $(1.8 \%)$, no mortality was reported after conversion.

An overview of all postoperative complications is depicted in Table 3. Median intensive care stay for uncomplicated patients was 2 days (IQR 1-3 days) and 3 days (IQR 1-9 days) for patients with a complicated postoperative course (all grades of complications) $(p=<0.001)$. Average hospital stay for patients with an uncomplicated postoperative course was 10 days (IQR 8-13 days) as compared to 23 days (IQR 12-41 days) for patients with a complicated postoperative course $(p=<0.001)$. Isolated pulmonary complications were observed in 37 patients $(13.1 \%)$, with a median hospital stay of 14 days (IQR 9.25-17 days).
Anastomotic leakage was observed in 43 patients (15.2\%). Using the classification proposed by Low et al., grade I leakage was observed in 6 patients $(2.1 \%)$, grade II in 8 patients $(2.8 \%)$, grade III in 16 patients $(5.7 \%)$ and grade IV in 13 patients (4.6\%) [13]. Grades and according hospital stay are depicted in Table 3. Comparison of linear versus circular stapling techniques depicted no differences in leakage rate, being 13 versus $14.9 \%$, respectively ( $p=0.710$ ).

Two patients developed a tracheoesophageal fistula following anastomotic leakage, treated with endoscopic stents in one patient and a reoperation in order to repair the fistula in the other patient. No differences were observed in leak rates between the participating hospitals $(p=0.334)$.

The effect of was determined using binary logistic regression analysis. None of the parameters were found to be predictive for anastomotic leak as depicted in Table 4. 
Table 3 Postoperative complications and frequencies

\begin{tabular}{lrrr}
\hline Complications description & $N(282)$ & $\%$ & $\begin{array}{c}\text { Hospital stay } \\
\text { median (IQR) }\end{array}$ \\
\hline Anastomotic leakage & 43 & 15.2 & \\
Grade I & 6 & 2.1 & $25(18-40)$ \\
Grade II & 8 & 2.8 & $32(29-92)$ \\
Grade III & 16 & 5.7 & $40(25-67)$ \\
Grade IV & 13 & 4.6 & $45(37-65)$ \\
Pulmonary complications & 37 & 13.1 & \\
Cardiovascular complications & 12 & 4.3 & \\
Wound infection & 9 & 3.5 & \\
Bronchoesophageal fistula & 2 & & \\
Other (9) & & & \\
Paraesophageal herniation & 3 & & \\
Bleeding & 2 & & \\
Reoperation for suspected torsion of gastric conduit & 1 & & \\
Reoperation for suspected anastomotic leakage & 1 & & \\
Leakage of staple line stomach & 1 & & \\
Iatrogenic lesion of spleen & 1 & \\
\hline
\end{tabular}

For the different grades of anastomotic leakage, the median (IQR) hospital stay is depicted, hospital stay increases with increasing grades of anastomotic leak, with Kruskal-Wallis one-way ANOVA for $k$ samples $p<0.001$
Six $(2.1 \%)$ patients died in hospital, or within 30-days postoperatively, having a Clavien-Dindo grade V complication. Four patients died after developing multiorgan failure due to sepsis following pneumonia. One patient died as a consequence of heart failure. The last patient died following hemorrhage from a tracheoesophageal fistula.

\section{Pathology}

A microscopically radical (R0) resection was obtained in $92.5 \%$ of patients. A complete pathologic response to neoadjuvant therapy was observed in $20.1 \%$ of patients after chemoradiotherapy. Average lymph node yield was 22.9 ( \pm 9.7$)$ lymph nodes.

\section{Discussion}

This multicenter pooled cohort study describes the initial results of 282 patients with distal esophageal and gastroesophageal junction adenocarcinomas, treated with neoadjuvant therapy, usually chemoradiotherapy, followed by a total minimally invasive Ivor Lewis esophagectomy [12]. The treatment resulted in a radical resection in $>90 \%$ of patients. Although the leakage rate was relatively high, extensive treatment resulted in a low combined in-hospital and 30-day mortality rate of $2.1 \%$, which is in concordance with other studies [5, 19]. According to these results, MIE-IL can be considered safe.
Table 4 Binary logistic regression analysis for effect of anastomotic technique, duration of surgery, neoadjuvant therapy and clinical $\mathrm{T}$ stage on the occurrence of anastomotic leak

\begin{tabular}{llllll}
\hline Parameter & $B$ & Sig. & $\operatorname{Exp}(B)$ & \multicolumn{2}{l}{$95 \%$ C.I. for EXP $(B)$} \\
\cline { 5 - 6 } & & & & Lower & Upper \\
\hline Hospital & & .216 & & & \\
Clinical T stage & -.512 & .350 & .599 & .205 & 8.753 \\
Neoadjuvant treatment (yes/no) & -.199 & .866 & .820 & .081 & 1.005 \\
Duration of surgery (min) & -.004 & .402 & .996 & .987 & 1.916 \\
Anastomosis (linear/circular) & -2.482 & .120 & .084 & .004 & \\
Constant & 1.126 & .591 & 3.083 & & \\
\hline
\end{tabular}

$p$ value of overall model being $p=0.104$ 
Previous studies have favored cervical anastomosis, reporting that cervical leakage was manageable with a cervical enterocutaneous fistula, with less morbidity and mortality compared to leakage following thoracic anastomosis [20]. There is evidence that cervical anastomoses are associated with a higher anastomotic leakage rate, more stenosis and more recurrent laryngeal nerve lesions, although available randomized evidence is limited [4]. A recent study showed comparable morbidity and mortality rates following cervical or thoracic anastomosis [21]. Differences in anastomotic leak rate may be explained by a shorter gastric tube segment in MIE-IL, possibly holding for better vascularization at the site of anastomosis [22].

Although our main conclusion is that MIE-IL can be considered safe, several observations should be addressed. Overall anastomotic leakage was observed in $15.2 \%$ of patients. However, the incidence of the higher grades was relatively low. The overall leakage rate observed in this study is in concordance with the available literature [19, 23-25]. Previous studies described an empyema rate of $5 \%$, an anastomotic leakage requiring surgery in $4 \%$ of patients and a gastric tube necrosis in $2 \%$ of patients [25].

No statistically significant differences in anastomotic leak rate were observed for the five different techniques used in this cohort, although it should be noted some techniques were not applied often, and statistical power for comparison of these techniques is low. Here, the general principles of anastomosis such as tension- and rotationfree, patency and optimal perfusion are essential. Intraoperative evaluation of the anastomosis may be performed with methylene blue or endoscopy, but evidence has not been obtained in a systematic manner. The use of omentoplasty covering the anastomosis resulted in less postoperative anastomotic leakage after esophagectomy in a Cochrane study, but the outcome after transthoracic anastomosis was just not found significant probably because of the low number of studies including this type of anastomosis [26].

Regression analysis determined that anastomotic technique, duration of surgery, neoadjuvant therapy and clinical $\mathrm{T}$ stage were not predictive for anastomotic leak. Further emphasizing different techniques may be considered safe, depending on local expertise.

Pulmonary complications were observed in $13.3 \%$, similar to the results observed in the TIME-trial, in which pulmonary complications were observed in $12 \%$ of patients in the minimally invasive group and $34 \%$ in patients operated in the open group [5]. Interestingly, most patients in this series were operated in prone position, whereas previous series have mainly operated in lateral decubitus position $[19,25]$. In the lateral approach, using selective intubation, a lung block is applied during the whole operation, whereas in prone position lung block is not necessary at all. Thoracoscopic surgery in prone position has shown to allow for earlier mobilization and less respiratory complications [27].

No recurrent nerve lesions were observed here in the present cohort. Previous studies displayed similar results with recurrent nerve lesions in $8 \%$ of patients with cervical anastomosis and $1 \%$ of patients with intrathoracic anastomosis [25].

Overall hospital stay and intensive care (ICU) stay were longer following postoperative complications, with an ICU stay of a median of 2 and 3 days and a median of 10 and 23 days for uncomplicated and complicated hospital stay, respectively. These results are in concordance with the literature stating average stays of 2 days for ICU and 7 days for hospital stay [25].

Indications for MIE-IL vary. Some surgeons use this approach for treating gastroesophageal junction tumors only, whereas other surgeons claim that for treating distal esophageal tumors, a safe resection can be performed with a margin of $5 \mathrm{~cm}$ and adequate subcarinal and paratracheal lymphadenectomy, thereby making this approach suitable for tumors located up to $5-\mathrm{cm}$ distal of the carina [19, 28, 29].

Over ninety percent of patients in this cohort received neoadjuvant therapy, usually consisting of chemoradiotherapy [6]. In previous series of MIE-IL, only $29 \%$ of patients received neoadjuvant therapy [25]. It has been stated that chemoradiotherapy might affect anastomotic healing [30]. Two series reported anastomotic complications in 6.7 and $13 \%$ of patients that received chemoradiotherapy followed by IL esophagectomy [30, 31]. Multivariable analysis found that the preoperative radiation dose received on the fundus of the stomach was associated with anastomotic complications. The radiation dose received by the proximal esophagus was not associated with anastomotic complications following IL [30].

In the CROSS study, no differences were found in predominantly cervical anastomotic leakage rates between patients that received neoadjuvant chemoradiotherapy versus patients that received no neoadjuvant therapy [6].

In conclusion, considering the increase in distal adenocarcinomas in the West and possible benefits on postoperative morbidity, the advantages of a two-stage procedure should be recognized: a shorter gastric tube segment accounting for better vascularization of the anastomosis, less recurrent nerve injuries and less stenosis compared to cervical anastomosis. Long-term oncological safety is to be determined in follow-up studies. Future research should address implementation problems, such as standardization of operative techniques and type of anastomosis. According to the IDEAL framework, this procedure is moving from the Development to the Exploration stage. The following stage will encompass a consensus in order to select 
the best (two) procedure (s) in order to perform a randomized controlled trial [32]. The primary goal of the study will be to decrease all postoperative complications, while maintaining optimal quality of surgical oncological resection.

\section{Compliance with ethical standards}

Disclosures Jennifer Straatman, Nicole van der Wielen, Grard A. P. Nieuwenhuijzen, Camiel Rosman, Josep Roig, Joris J. G. Scheepers, Miguel A. Cuesta, Misha D. P. Luyer, Mark I. van Berge Henegouwen, Frans van Workum, Suzanne S. Gisbertz and Donald L. van der Peet have no conflicts of interest or financial ties to disclose.

Open Access This article is distributed under the terms of the Creative Commons Attribution 4.0 International License (http://crea tivecommons.org/licenses/by/4.0/), which permits unrestricted use, distribution, and reproduction in any medium, provided you give appropriate credit to the original author(s) and the source, provide a link to the Creative Commons license, and indicate if changes were made.

\section{References}

1. Lewis I (1946) The surgical treatment of carcinoma of the esophagus: with special reference to a new operation for growths of the middle third. Br J Surg 34:18-31

2. McKeown KC (1976) Total three-stage esophagectomy for cancer of the esophagus. Br J Surg 63:259-262

3. Scheepers JJ, van der Peet DL, Veenhof AA, Heijnen B, Cuesta MA (2009) Systematic approach of postoperative gastric conduit complications after esophageal resection. Dis Esophagus 23:117-121

4. Biere SS, Maas KW, Cuesta MA, van der Peet DL (2011) Cervical or thoracic anastomosis after esophagectomy for cancer: a systematic review and meta-analysis. Dig Surg 28:29-35

5. Biere SS, van Berge Henegouwen MI, Maas KW, Bonavina L, Rosman C, Garcia JR, Gisbertz SS, Klinkenbijl JH, Hollmann MW, de Lange ES, Bonjer HJ, van der Peet DL, Cuesta MA (2012) Minimally invasive versus open oesophagectomy for patients with oesophageal cancer: a multicentre, open-label, randomised controlled trial. Lancet 379:1887-1892

6. van Hagen P, Hulshof MC, van Lanschot JJ, Steyerberg EW, van Berge Henegouwen MI, Wijnhoven BP, Richel DJ, Nieuwenhuijzen GA, Hospers GA, Bonenkamp JJ, Cuesta MA, Blaisse RJ, Busch OR, ten Kate FJ, Creemers GJ, Punt CJ, Plukker JT, Verheul HM, Spillenaar Bilgen EJ, van Dekken H, van der Sangen MJ, Rozema T, Biermann K, Beukema JC, Piet AH, van Rij CM, Reinders JG, Tilanus HW, van der Gaast A, Group C (2012) Preoperative chemoradiotherapy for esophageal or junctional cancer. N Engl J Med 366:2074-2084

7. Scheepers JJ, Mulder CJ, Van Der Peet DL, Meijer S, Cuesta MA (2006) Minimally invasive oesophageal resection for distal oesophageal cancer: a review of the literature. Scand J Gastroenterol Suppl 243:123-134

8. Palanivelu C, Prakash A, Senthilkumar R, Senthilnathan P, Parthasarathi R, Rajan PS, Venkatachlam S (2006) Minimally invasive esophagectomy: thoracoscopic mobilization of the esophagus and mediastinal lymphadenectomy in prone positionexperience of 130 patients. J Am Coll Surg 203:7-16

9. Luketich JD, Alvelo-Rivera M, Buenaventura PO, Christie NA, McCaughan JS, Litle VR, Schauer PR, Close JM, Fernando HC
(2003) Minimally invasive esophagectomy: outcomes in 222 patients. Ann Surg 238:494-495

10. Biere SS, Cuesta MA, van der Peet DL (2009) Minimally invasive versus open esophagectomy for cancer: a systematic review and meta-analysis. Minerva Chir 64:121-133

11. Nagpal K, Ahmed K, Vats A, Yakoub D, James D, Ashrafian H, Darzi A, Moorthy K, Athanasiou T (2010) Is minimally invasive surgery beneficial in the management of esophageal cancer? A meta-analysis. Surg Endosc 24:1621-1629

12. Siewert JR, Stein HJ, Feith M (2006) Adenocarcinoma of the esophagogastric junction. Scand J Surg 95:260-269

13. Low DE, Alderson D, Cecconello I, Chang AC, Darling GE, D'Journo XB, Griffin SM, Holscher AH, Hofstetter WL, Jobe BA, Kitagawa Y, Kucharczuk JC, Law SY, Lerut TE, Maynard N, Pera M, Peters JH, Pramesh CS, Reynolds JV, Smithers BM, van Lanschot JJ (2015) International consensus on standardization of data collection for complications associated with esophagectomy: Esophagectomy Complications Consensus Group (ECCG). Ann Surg 262(2):286-294

14. Clavien PA, Barkun J, de Oliveira ML, Vauthey JN, Dindo D, Schulick RD, de Santibañes E, Pekolj J, Slankamenac K, Bassi C, Graf R, Vonlanthen R, Padbury R, Cameron JL, Makuuchi M (2009) The Clavien-Dindo classification of surgical complications: five year experience. Ann Surg 250:187-196

15. Takubo K, Vieth M, Aida J, Sawabe M, Kumagai Y, Hoshihara Y, Arai T (2009) Differences in the definitions used for esophageal and gastric diseases in different countries: endoscopic definition of the esophagogastric junction, the precursor of Barrett's adenocarcinoma, the definition of Barrett's esophagus, and histologic criteria for mucosal adenocarcinoma or high-grade dysplasia. Digestion 80:248-257

16. Japanese Esophageal Society (2009) Japanese classification of esophageal cancer; tenth edition-part 1. Esophagus 6:1-25

17. Maas KW, Biere SS, Scheepers JJ, Gisbertz SS, Turrado Rodriguez VT, van der Peet DL, Cuesta MA (2012) Minimally invasive intrathoracic anastomosis after Ivor Lewis esophagectomy for cancer: a review of transoral or transthoracic use of staplers. Surg Endosc 26:1795-1802

18. van Workum F, van den Wildenberg FJ, Polat F, de Wilt JH, Rosman C (2014) Minimally invasive oesophagectomy: preliminary results after introduction of an intrathoracic anastomosis. Dig Surg 31:95-103

19. Nguyen NT, Hinojosa MW, Smith BR, Chang KJ, Gray J, Hoyt D (2008) Minimally invasive esophagectomy lessons learned from 104 operations. Ann Surg 248:1081-1091

20. Huang HT, Wang F, Shen L, Xia CQ, Lu CX, Zhong CJ (2015) Clinical outcome of middle thoracic esophageal cancer with intrathoracic or cervical anastomosis. Thorac Cardiovasc Surg 63(4):328-334

21. Shah DR, Martinez SR, Canter RJ, Yang AD, Bold RJ, Khatri VP (2013) Comparative morbidity and mortality from cervical or thoracic esophageal anastomoses. J Surg Oncol 108:472-476

22. Klink CD, Binnebosel M, Otto J, Boehm G, von Trotha KT, Hilgers RD, Conze J, Neumann UP, Jansen M (2012) Intrathoracic versus cervical anastomosis after resection of esophageal cancer: a matched pair analysis of 72 patients in a single center study. World J Surg Oncol 10:159

23. Xie MR, Liu CQ, Guo MF, Mei XY, Sun XH, Xu MQ (2014) Short-term outcomes of minimally invasive Ivor-Lewis esophagectomy for esophageal cancer. Ann Thorac Surg 97:1721-1727

24. Sihag S, Wright CD, Wain JC, Gaissert HA, Lanuti M, Allan JS, Mathisen DJ, Morse CR (2012) Comparison of perioperative outcomes following open versus minimally invasive Ivor Lewis oesophagectomy at a single, high-volume centre. Eur J CardioThorac Surg 42:430-437 
25. Luketich JD, Pennathur A, Awais O, Levy RM, Keeley S, Shende M, Christie NA, Weksler B, Landreneau RJ, Abbas G, Schuchert MJ, Nason KS (2012) Outcomes after minimally invasive esophagectomy: review of over 1000 patients. Ann Surg 256:95-103

26. Yuan Y, Zeng X, Hu Y, Xie T, Zhao Y (2014) Omentoplasty for oesophagogastrostomy after oesophagectomy. Cochrane Database Syst Rev 10:CD008446

27. Yatabe T, Kitagawa H, Yamashita K, Hanazaki K, Yokoyama M (2013) Comparison of the perioperative outcome of esophagectomy by thoracoscopy in the prone position with that of thoracotomy in the lateral decubitus position. Surg Today 43:386-391

28. Ribet M, Debrueres R, Iecomte HM (1992) Resection for advanced cancer of the thoracic esophagus: cervical or thoracic anastomosis? Late results of a prospective randomized study. J Thorac Cardiovasc Surg 103:784-789

29. Sutton CD, White SA, Marshall LJ, Berry DP, Veitch PS (2002) Endoscopic-assisted intrathoracic oesophagogastrostomy without thoracotomy for tumours of the lower oesophagus and cardia. Eur J Surg Oncol 28:46-48

30. Vande Walle C, Ceelen WP, Boterberg T, Vande Putte D, Van Nieuwenhove Y, Varin O, Pattyn P (2012) Anastomotic complications after Ivor Lewis esophagectomy in patients treated with neoadjuvant chemoradiation are related to radiation dose to the gastric fundus. Int J Radiat Oncol Biol Phys 82:e513-e519

31. Thomay AA, Snyder JA, Edmondson DM, Scott WJ (2012) Initial results of minimally invasive Ivor Lewis esophagectomy after induction chemoradiation (50.4 gy) for esophageal cancer. Innovations 7:421-428

32. McCulloch P, Altman DG, Campbell WB, Flum DR, Glasziou P, Marshall JC, Nicholl J, Balliol C, Aronson JK, Barkun JS, Blazeby JM, Boutron IC, Campbell WB, Clavien PA, Cook JA, Ergina PL, Feldman LS, Flum DR, Maddern GJ, Nicholl J, Reeves BC, Seiler CM, Strasberg SM, Meakins JL, Ashby D, Black N, Bunker J, Burton M, Campbell M, Chalkidou K, Chalmers I, de Leval M, Deeks J, Ergina PL, Grant A, Gray M, Greenhalgh R, Jenicek M, Kehoe S, Lilford R, Littlejohns P, Loke Y, Madhock R, McPherson K, Meakins J, Rothwell P, Summerskill B, Taggart D, Tekkis P, Thompson M, Treasure T, Trohler U, Vandenbroucke J (2009) No surgical innovation without evaluation: the IDEAL recommendations. Lancet 374:1105-1112 